\title{
Formaldehyde Exposure Induces Differentiation of Regulatory T Cells via the NFAT- mediated T Cell Receptor Signalling Pathway in Yucatan Minipigs
}

Jeongsik Park

Korea Institute of Toxicology

Goo-Hwa Kang

Korea Institute of Toxicology

Youngkyu Kim

Korea Institute of Toxicology

Ju Young Lee

Korea Institute of Toxicology

Jeong Ho Hwang ( $\boldsymbol{\sim}$ jeongho.hwang@kitox.re.kr)

Korea Institute of Toxicology

\section{Research Article}

Keywords: Formaldehyde exposure, NFAT- mediated, minipigs (Sus scrofa), toxicological and pharmacological research

Posted Date: August 26th, 2021

DOl: https://doi.org/10.21203/rs.3.rs-841996/v1

License: (c) (1) This work is licensed under a Creative Commons Attribution 4.0 International License. Read Full License 


\section{Abstract}

The use of minipigs (Sus scrofa) as a platform for toxicological and pharmacological research is well established. In the present study, we investigated the effect of formaldehyde (FA) exposure on helper T cell-mediated splenic immune responses in Yucatan minipigs. The minipigs were exposed to different concentrations of FA $\left(0,2.16,4.62\right.$, or $\left.10.48 \mathrm{mg} / \mathrm{m}^{3}\right)$ for a period of 2 weeks. Immune responses elicited by exposure to FA were determined by assessing physiological parameters, mRNA expression, and cytokine production. Additionally, the distribution of helper T cells and regulatory T cells (Treg) and expression of NFAT families, which are well-known T cell receptor signalling proteins associated with regulatory T cell development, were evaluated. Exposure to FA suppressed the expression of genes associated with Th1 and Th2 cells in minipigs in a dose-dependent manner. Subsequent production of cytokines also declined post-FA exposure. Furthermore, exposure to FA induced the differentiation of $\mathrm{CD}^{+}{ }^{+}$Foxp $^{+}{ }^{+}$Treg cells with divergent expression levels of NFAT1 and NFAT2. These results indicated that exposure to FA increased the Treg cell population via the NFAT-mediated T cell receptor signalling pathway, leading to suppression of the effector T cell activity with a decline in T cell-related cytokine production.

\section{Introduction}

Minipigs (Sus scrofa) have been extensively reported for their value and utility as an animal model for research ${ }^{[1]}$. In the EU, to date, more than 60,000 minipigs are used every year for scientific research, and they are a well-established animal model for surgical, physiological, and biomedical research ${ }^{\text {[2] }}$. There has been a notable interest in minipigs due to the anatomical, physiological, and biochemical similarities between minipigs and humans ${ }^{[1-4]}$, particularly in terms of the nasal cavity, skin, and heart ${ }^{[2-3]}$. Several sequence studies have reported that the evolutionary distance between pig and human is smaller than that between rodent and human ${ }^{[5-7]}$, indicating that the pig genome is fairly homologous with the human genome. Recently, several human diseases that could not be reflected in a rodent model have been successfully modelled in pigs through genetic modification ${ }^{[8-9]}$. Additionally, the use of minipigs in toxicological and pharmacological research, including in the analysis of pulmonary exposure to chemicals and drugs, has recently been reported ${ }^{[10-13]}$. Thus, minipigs are a useful non-rodent animal model for use in toxicology and pharmacology studies.

Formaldehyde (FA), a colourless, flammable, strongly reactive chemical present in homes and other buildings, is a common indoor and outdoor pollutant ${ }^{[14]}$. FA is a common component of pressed-wood products, urea-FA insulations, and glues and adhesives ${ }^{[15]}$, and is also widely present in plastics, cosmetics, industrial fungicides, and disinfectants [16]. Thus, individuals can be subjected to high levels of FA via inhalation at their homes, workplaces, etc. Additionally, FA is recognized as a toxic chemical at certain doses (80-2000 ppb) and the health risks are increased at room temperature due to the volatile nature of FA ${ }^{[17-18]}$. The International Agency for Research on Cancer has classified FA as a category-1 human and animal carcinogen responsible for nasopharyngeal cancer and leukaemia ${ }^{[19-20]}$. According 
to a toxicological review by the US Environmental Protection Agency, FA induces adverse events, including sensory irritation and immuno-, neuro-, and developmental-toxicity ${ }^{[21]}$. Recent human and animal studies have reported that immune dysregulation caused by FA exposure may aggravate allergic inflammation ${ }^{[22-23]}$. Conversely, immunosuppression caused by FA exposure may lead to cancer progression ${ }^{[24-26]}$. These studies indicate that FA exerts potential toxic effects on the immune system.

The association between FA exposure and asthma has been investigated by several studies ${ }^{[17,27-31]}$. Jung et al. showed that exposure to FA induces and aggravates airway inflammation by promoting eosinophil infiltration and T cell-related cytokine production ${ }^{[32]}$. Conversely, recent studies have indicated that FA-exposed rodents sensitized with ovalbumin exhibited impaired development of allergic responses, along with reduced $T$ cell-related cytokine production, bronchial responsiveness, and mast cell activation $[17,27]$. FA also affects the different types of $T$ cells, including $C D 4^{+} T$ cells, $C D 8^{+} T$ cells, and memory $T$ cells ${ }^{[33-35]}$. Sandikci et al. reported that the levels of $\mathrm{CD} 4^{+}$and $\mathrm{CD} 8^{+} T$ cells were increased in adult rats

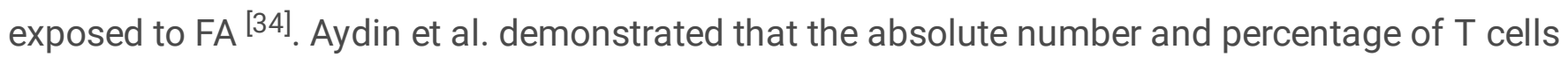

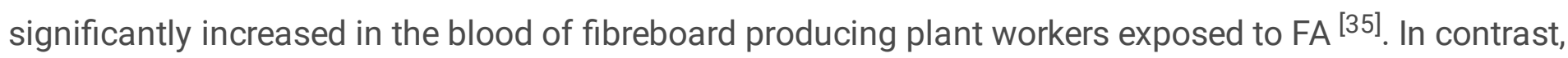
Hosgood et al. reported that the counts of $\mathrm{CD} 4^{+}$and $\mathrm{CD} 8^{+} \mathrm{T}$ cell were significantly low in workers

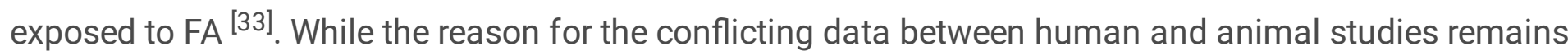
unclear, previous reports have, overall, stressed the need for further research assessing the potential toxic effects of FA exposure on the immune system.

$\mathrm{CD} 4^{+}$helper T cells play crucial roles in host health and immune-mediated disease ${ }^{[36]}$. They can differentiate into different subsets of effector T cells (Th1 and Th2) and regulatory T (Treg) cells after activation ${ }^{[37]}$. The three subsets express distinct cytokine signatures, master transcription factors, and homing receptors in response to infection by pathogens ${ }^{[36]}$. Th1 cells are characterized by the expression of interferon- $\gamma$ (IFN- $\gamma$ ), interleukin-2 (IL-2), and tumour necrosis factor-a (TNF-a) ${ }^{[37]}$. These cells are particularly important in the defence against intracellular bacteria, such as Listeria and Mycobacterium tuberculosis, but can also exacerbate the development of organ-specific autoimmune diseases and chronic inflammatory disorders ${ }^{[38]}$. Th2 cells express IL-4, IL-5, and IL-13 and are immunologically active against extracellular pathogens, such as worms ${ }^{[39]}$. Th2 cells also promote acute and chronic inflammatory responses against a myriad of allergens ${ }^{[40]}$. Treg cells are characterized by the expression of the forkhead transcription factor, Foxp3, which is essential for their development and suppressive functions ${ }^{[41]}$. These cells play a critical role in maintaining the homeostasis of the immune system, regulating effector $T$ cell responses, and preventing autoimmune reactivity ${ }^{[42]}$. However, an excessive presence of Treg cells can have a detrimental effect on the host due to potentially increasing susceptibility to opportunistic infections and inhibition of antitumor immunity [43-45].

Several studies have investigated the relationship between FA exposure and immune responses in rodent model systems. However, FA exposure studies using minipigs as a non-rodent animal model have not yet been reported. In the present study, we used Yucatan minipigs to investigate the effects of FA exposure 
on the immune system by evaluating the helper $T$ cell and Treg cell populations and the expression of immune-related factors, including cytokines. Therefore, the aim of this study is to offer insight into the underlying mechanism orchestrating the FA-exposure-induced immune modulation that has detrimental health effects, such as opportunistic infections and cancer development.

\section{Methods}

\subsection{Animal}

Six-month-old male Yucatan minipigs (Optipharm Inc., Chenongju, Korea) were used in this study. Each minipig was placed in an individual pen in an animal room with controlled temperature $\left(22 \pm 2^{\circ} \mathrm{C}\right)$ and humidity (50 $\pm 5 \%$ ), a $12 \mathrm{~h}$ light/dark cycle, and positive pressure with HEPA-filtered air. The animals were fed sterilized food pellets (Farm story Dodram B\&F, Seoul, Korea) and sterilized tap water ad libitum and were acclimatized for 3 weeks prior to commencement of FA exposure. All experimental procedures carried out in this study were reviewed and approved by the Institutional Animal Care and Use Committee of the Korea Institute of Toxicology (IACUC \#1908 - 0297) and were performed in accordance with the relevant guidelines and regulations.

\subsection{Experimental group}

The minipigs were randomly divided into four groups ( $n=2$ minipigs/group): control group, $2.16 \pm 0.16$ $\mathrm{mg} / \mathrm{m}^{3} \mathrm{FA}$ exposure group, $4.62 \pm 0.36 \mathrm{mg} / \mathrm{m}^{3} \mathrm{FA}$ exposure group, and $10.48 \pm 0.64 \mathrm{mg} / \mathrm{m}^{3} \mathrm{FA}$ exposure group. The control minipigs were not exposed to FA. Minipigs in the treatment groups were exposed to FA for a period of 2 weeks at $2 \mathrm{~h}$ /day for 5 days a week through a minipig mask type inhalation system. Body weights were measured on days 2, 6, 9, and 13 prior to FA exposure. Terminal body weight was measured $24 \mathrm{~h}$ after the last FA exposure. The pigs were sedated using $0.5 \mathrm{mg} / \mathrm{kg}$ midazolam and 5 $\mathrm{mg} / \mathrm{kg}$ ketamine intramuscularly and were sacrificed under isoflurane anaesthesia. All samples were collected for subsequent analysis.

\subsection{FA exposure}

FA was administered via a methanol-free ultrapure $10 \%$ FA solution (Polysciences Inc., Warrington, PA, USA) using the minipig mask type inhalation system. The FA was diluted with clean air using a multi-neck flask to achieve the desired FA concentrations and delivered through the minipig mask. The FA in the minipig mask was sampled using a Top Solid DNPH Cartridge (Top-Trading Co., Seoul, Korea) and was monitored hourly by high-performance liquid chromatography with ultraviolet detection (HPLC-UV).

\subsection{HPLC-UV analysis}

The FA-2,4-dinitrophenylhydrazine derivative was analysed on an Agilent 1260 Infinity HPLC system (Agilent Technologies, Santa Clara, CA, USA) equipped with a degasser (Agilent 1260 Infinity series, G1322A), a binary pump (Agilent 1260 Infinity series, G1312B), an autosampler (Agilent 1260 Infinity series, G1329B), a thermostat column compartment (Agilent 1260 Infinity series, G1316B), and a diode 
array detector VL (Agilent 1260 Infinity series, G1315D). A Gemini $5 \mu \mathrm{mC18}$ 110A column (length, 150 $\mathrm{mm}$; diameter, $4.6 \mathrm{~mm}$; particle size, $5 \mu \mathrm{m}$; Phenomenex, Torrance, CA, USA) was used as an analytical column. Acetonitrile and distilled water $(60: 40, \mathrm{v} / \mathrm{v})$ were used as the mobile phase. The injection volume was $10 \mu \mathrm{L}$, flow rate was $1.0 \mathrm{~mL} / \mathrm{min}$, and column temperature was $40^{\circ} \mathrm{C}$. The analyte was monitored at a wavelength of $360 \mathrm{~nm}$. Quantitation was performed using a synthesized FA-2,4-DNPH solution (SigmaAldrich Co., St. Louis, MO, USA) as the standard.

\subsection{Spleen cell preparation and culture}

The spleen was isolated from each minipig exposed to FA. Single splenic cells were prepared as described previously ${ }^{[46]}$. For primary cell culture, the splenic single cells were suspended in RPMI 1640 media (Lonza, Walkersville, MD, USA) containing $10 \%$ heat inactivated foetal bovine serum (Gibco Laboratory, Grand Island, NY, USA), penicillin (100 U/mL) (Gibco Laboratory), and streptomycin (100 $\mu \mathrm{g} / \mathrm{mL}$ ) (Gibco Laboratory). Splenocytes were seeded at a density of $1 \times 10^{6}$ cells $/ 100 \mu \mathrm{L} /$ well in 12 well culture plates (Eppendorf, Hamburg, AG, Germany) and incubated for $72 \mathrm{~h}$ with $2.5 \mu \mathrm{g} / \mathrm{mL}$ Concanavalin $\mathrm{A}$ (Con A; Sigma-Aldrich Co.) at $37^{\circ} \mathrm{C}$ with $5 \% \mathrm{CO}_{2}$.

For flow cytometric analysis, the splenic single cells were re-suspended in Flow Cytometry Staining Buffer (eBioscience Inc., San Diego, CA, USA) prior to analysis.

\subsection{Quantitative real time-polymerase chain reaction (qRT- PCR)}

Total RNA was extracted from cultured splenocytes using the RNeasy Mini Kit (Qiagen, Valencia, CA, USA) according to the manufacturer's protocol and quantified using a QIAxpert system (Qiagen). Complementary DNA was synthesized by reverse transcribing total RNA (400 ng) using the GoScript ${ }^{\text {TM }}$ Reverse Transcription system (Promega, Madison, WI, USA) in MasterCycler® Nexus GX2 (Eppendorf). The primer sequences were as follows; minipig GAPDH, 5'-GGGCATGAACCATGAGAAGT-3' and 5'TGTGGTCATGAGTCCTTCCA-3', IL-4, 5'-AGAACACGACGGAGAAGGAA-3' and 5'-TTGCCATGCTGCTAGGTT3'; IFN- $y$, 5'-CAGCTTTGCGTGACTTTGTG-3' and 5'-TTTTGTCACTCTCCTTCCAAT-3'; and TNF- $a$, 5'CCCCCAGAAGGAAGAGTTTC-3' and 5'-CGGGCTTATCTGAGGTTTGA-3'. The qRT-PCR was performed on the QuantStudio ${ }^{\text {TM }} 5$ Real-Time system (Thermo Scientific, Wilmington, DE, USA) with the Power SYBR Green Master Mix (Thermo Scientific). The transcript level for each gene was normalized to GAPDH.

\subsection{Cytokine production of spleen cell culture supernatant}

The splenocyte culture supernatants were assessed for the cytokines IL-4, IFN- - , and TNF-a using the ProcartaPlex ${ }^{\mathrm{TM}}$ immunoassay kit (eBioscience) and the Luminex $200^{\mathrm{TM}}$ system (Luminex Corporation, Austin, TX, USA) according to the manufacturer's instructions. Cytokine concentrations were quantified by evaluating the fluorescent signal of analyte-specific capture beads and analysed using ProcartaPlex Analyst 1.0 (eBioscience). All standards and samples were measured in duplicates.

\subsection{Flow cytometric analysis}


The single spleen cells isolated from FA exposed minipigs were washed with Flow Cytometry Staining Buffer (eBioscience) and stained with PC 5.5-conjugated anti-CD4 monoclonal antibody (BD Biosciences, San Jose, CA, USA) for 60 min on ice. For intracellular staining, the spleen cells were permeabilized with a Fixation/Permeablization solution (eBioscience) and stained with an FITC-conjugated anti-Foxp3 monoclonal antibody (eBioscience) for $60 \mathrm{~min}$ at $20^{\circ} \mathrm{C}$. All samples were run on CytoFlex Flow Cytometer (Thermo Scientific). Data of 50,000 events were collected and analysed using the CytoExpert 2.3 software (Thermo Scientific).

\subsection{Western blot analysis}

The spleen tissue was processed to generate denatured protein lysate in RIPA buffer (Pierce Biotechnology, Rockford, IL, USA), which was quantified using the Pierce BCA Protein Assay kit (Thermo Scientific). Protein lysates $(30 \mu \mathrm{g})$ were mixed with $4 \times$ Laemmli sample buffer (Bio-Rad Laboratories, Hercules, CA, USA), separated by $8 \%$ SDS-PAGE, and transferred onto polyvinylidene difluoride membranes (Millipore, Billerica, MA, USA). The membranes were blocked using $5 \%$ bovine serum albumin in Tris-buffered saline with $0.1 \%$ Tween 20 (TBS-T) for $60 \mathrm{~min}$ at $20-24^{\circ} \mathrm{C}$ The membranes were then incubated with anti-NFAT1, anti-NFAT2 (Abcam, Cambridge, UK), and anti- $\beta$-actin antibodies (Santa Cruz Biotechnology, Santa Cruz, CA, USA) overnight at $4^{\circ} \mathrm{C}$. After washing thrice with TBS-T, the membranes were incubated for $60 \mathrm{~min}$ at $20-24^{\circ} \mathrm{C}$ with horseradish peroxidase-conjugated goat antirabbit and anti-mouse IgG (Santa Cruz Biotechnology). After washing thrice with TBS-T, the immunoreactive signals were visualized with chemiluminescent reagents (Pierce Biotechnology) using the ChemiDoc MP Imaging system (BD Biosciences). The densitometrical analysis results for specific protein signals were quantified using Image $\mathrm{J} 1.51 \mathrm{~K}$ (National Institute of health, Bethesda, ND, USA). All results were normalized to the expression of $\beta$-actin.

\subsection{Statistics}

All data are presented as the mean \pm SD. Statistical analysis was performed using the Statistical Package for the Social Sciences (Version 25; SPSS, Chicago, IL, USA). Differences between groups were evaluated by one-way ANOVAs and Kruskal-Wallis test. Tukey's tests and Bonferroni-adjusted Mann-Whitney U-tests were used as the post-hoc tests. $p<0.05$ was considered statistically significant.

\section{Results}

\subsection{FA exposure does not induce a change in body or organ weight}

Exposure to FA did not precipitate any differences in the body weights $(p>0.918)$ of or any weight gain ( $p$ $>0.444$ ) in the minipigs in the FA-exposed group (Fig. 1a). In the control group exposed to clean air, the body weights increased from $10.1 \pm 1.6$ to $11.7 \pm 1.6 \mathrm{~kg}$. All animals in the FA-exposed group exhibited similar changes in body weight with time as those in the control group. We also assessed the effect of FA exposure on the relative organ weight in minipigs in the following manner. 


\section{Relative organ weight $=$ organ weight $/$ brain weight $\times 100$ $\%$.}

There were no significant changes in the relative weights of organs, including the lung, spleen, and thymus; lung (control group: $161.0 \pm 24.0 \%, 2.16 \mathrm{mg} / \mathrm{m}^{3}$ FA exposure group: $176.4 \pm 7.6 \%, 4.62 \mathrm{mg} / \mathrm{m}^{3}$ FA exposure group: $176.7 \pm 4.6 \%, 10.48 \mathrm{mg} / \mathrm{m}^{3}$ FA exposure group: $180.5 \pm 21.5 \%, p>0.675$ ); spleen (control group: $68.9 \pm 37.7 \%, 2.16 \mathrm{mg} / \mathrm{m}^{3} \mathrm{FA}$ exposure group: $50.5 \pm 24.2 \%, 4.62 \mathrm{mg} / \mathrm{m}^{3} \mathrm{FA}$ exposure group: $59.2 \pm 24.0 \%, 10.48 \mathrm{mg} / \mathrm{m}^{3} \mathrm{FA}$ exposure group: $54.3 \pm 6.0 \%, p>0.884$ ); and thymus (control group: $45.2 \pm 12.7 \%, 2.16 \mathrm{mg} / \mathrm{m}^{3}$ FA exposure group: $32.5 \pm 1.4 \%, 4.62 \mathrm{mg} / \mathrm{m}^{3} \mathrm{FA}$ exposure group: 44.5 $\pm 10.6 \%, 10.48 \mathrm{mg} / \mathrm{m}^{3}$ FA exposure group: $32.0 \pm 14.9 \%, p>0.668$ ) (Fig. 1b).

\subsection{FA exposure causes decline in IFN- $y$, TNF- $a$, and IL-4 production}

To investigate the effect of FA exposure on the immune system, we assessed the expression of genes associated with Th1 (IFN- $\gamma$, TNF- $a$ ) and Th2 (IL-4) cells and the subsequent production of their cytokines. Exposure to FA resulted in a decline in IFN- $y, T N F-a$, and $I L-4$ mRNA levels in a dose-dependent manner, with significant suppression of $I L-4$ expression observed at all assessed doses. Additionally, gene expression in the animals exposed to $10.48 \pm 0.64 \mathrm{mg} / \mathrm{m}^{3} \mathrm{FA}$ was significantly lower than that in the control group animals (Fig. 2a). A similar expression pattern was observed in the production of Th1- and Th2-related cytokines; however, the production of IL-4 $(p=0.02)$ and TNF- $\mathrm{a}(p=0.02)$ in the FA-exposure groups was significantly lower than that in the control group (Fig. 3).

\subsection{FA exposure promotes an increase in Treg cell population}

Exposure of minipigs to FA precipitated no significant differences in the $\mathrm{CD} 4^{+}$helper $\mathrm{T}$ cell population size relative to that in the control group (control group: $18.38 \%, 2.16 \mathrm{mg} / \mathrm{m}^{3} \mathrm{FA}$ exposure group: $20.12 \%$, $4.62 \mathrm{mg} / \mathrm{m}^{3}$ FA exposure group: $17.14 \%, 10.48 \mathrm{mg} / \mathrm{m}^{3}$ FA exposure group: $\left.16.94 \%, p=0.443\right)$. However, the Treg cell population ( $\mathrm{CD} 4^{+} \mathrm{Foxp}^{+}$cells) was found to be significantly increased from $3.26 \%$ (control) to $5.40 \%$ and $4.43 \%$ following exposure to $4.62 \mathrm{mg} / \mathrm{m}^{3}$ and $10.48 \mathrm{mg} / \mathrm{m}^{3}$ of FA, respectively (Fig. 4).

\subsection{FA exposure results in increased NFAT1 expression and a decrease in NFAT2 expression}

Nuclear factor of activated T cells (NFAT) families are well-known T cell receptor (TCR) signalling proteins related to key regulation of Treg cell development ${ }^{[42]}$. To investigate the molecular mechanism of Treg cells in T cell-related cytokine suppression due to FA exposure, we evaluated the protein expression of NFAT1 and NFAT2. Notably, NFAT1 expression in the $4.62 \mathrm{mg} / \mathrm{m}^{3}$ FA-exposure group was nearly 1.28 -fold higher than that in the control group. In contrast, NFAT2 expression in the FA-exposure groups was decreased by approximately 0.94 -fold compared with that in the control group. 


\section{Discussion}

The minipigs, which bear close similarity to humans, are a useful non-rodent animal model for toxicology research studies. This is the first study on minipigs addressing the issue of FA-exposure-induced immune modulation. Many studies have reported that FA exposure affects helper T cell-related immune responses. However, the role of Treg cells in FA-exposure-induced immune responses is still not wellunderstood.

FA is a ubiquitous environmental pollutant and its ingestion by inhalation constitutes occupational and environmental health hazards ${ }^{[47]}$. In the present study, we report the suppressive effects of FA exposure on splenic immune responses, determined by evaluating the helper $T$ cell and Treg cell populations and by assessing the expression of immune-related factors including mRNAs, cytokines, and proteins in Yucatan minipigs. We administered FA at a concentration of $2.16 \mathrm{mg} / \mathrm{m}^{3}$ in this study based on the noobserved-adverse-effect concentration (for mouse: $2.46 \mathrm{mg} / \mathrm{m}^{3}$, for monkey: $1.23 \mathrm{mg} / \mathrm{m}^{3}$ ) assigned by the Organisation for Economic Co-operation and Development Screening Information Dataset (OECD SIDS) ${ }^{[48]}$. The current short-term exposure limit for FA in the United States is $2.46 \mathrm{mg} / \mathrm{m}^{3}{ }^{[49]}$. We also assessed the effect of FA at a concentration of $4.62 \mathrm{mg} / \mathrm{m}^{3}$, as it was the lowest observed adverse effect concentration (for mice: $5.04 \mathrm{mg} / \mathrm{m}^{3}$, for monkey: $3.69 \sim 7.38 \mathrm{mg} / \mathrm{m}^{3}$ ) as determined by the OECD SIDS [48]. Finally, a concentration of $10.48 \mathrm{mg} / \mathrm{m}^{3} \mathrm{FA}$ was used as the optimal high concentration to continuously control the minipig inhalation system. A previous animal study using these concentrations reported that FA exposure impaired the function and differentiation of Natural Killer cells ${ }^{[50]}$. Other studies have also shown that FA exposure affects immune responses, including helper $T$ cells and lung inflammation-related up- and down-regulating the expression of genes and proteins ${ }^{[32,51,52]}$. Based on these reports and studies, we exposed minipigs to $2.16 \mathrm{mg} / \mathrm{m}^{3}, 4.62 \mathrm{mg} / \mathrm{m}^{3}$, and $10.48 \mathrm{mg} / \mathrm{m}^{3}$ for 2 weeks at $2 \mathrm{~h} /$ day and 5 days a week.

To investigate the effect of FA exposure on physiological parameters, the body weights and relative organ weights (including the lung, spleen, and thymus) of the Yucatan minipig exposed to FA were assessed. Our data showed that exposure to FA precipitated no difference in body weight, weight gain, and relative weight of various organs. These findings are consistent with those of previous studies ${ }^{[30,32]}$. Additionally, to examine the effect of FA exposure on airway inflammation, the total and differential cell counts of macrophages, eosinophils, neutrophils, and lymphocytes in the bronchoalveolar lavage fluid were determined and histopathological analyses were conducted (data not shown). No significant differences were observed between the total and differential cell counts of the FA-exposed- and control-groups. These findings are consistent with those of previous studies showing that exposure to FA does not induce any significant differences in the counts of the various types of inflammatory cells in the bronchoalveolar lavage fluid ${ }^{[30,46]}$. However, in the histopathological analysis, infiltration of inflammatory cells and degeneration of the bronchial epithelium were noted to be increased in the $10.48 \mathrm{mg} / \mathrm{m}^{3}$ FA-exposure group. These observations suggested that the FA doses used in this study caused minimal airway 
inflammation but did not induce direct lung injury. Thus, in the present study, we investigated changes in the splenic immune response at FA concentrations that did not directly promote lung injury.

The spleen is a highly organized lymphoid organ and is very important for the innate and adaptive immune response ${ }^{[53]}$. In the spleen, the proper differentiation and development of different subsets of effector T cells (Th1, Th2) and Treg cells are initiated in the presence of lineage-specific effector cytokines during $T$ cell activation ${ }^{[54]}$. Recent human and animal studies have reported that FA exposure adversely affects the immune system by altering the population of different types of $T$ cells, as well as the production of helper T cell-related cytokines ${ }^{[29,33-35,46]}$. Thus, we investigated the potential effect of FA exposure on splenic immune responses by evaluating the expression of helper T cell-related mRNAs and cytokines. Our results demonstrate that FA exposure suppressed the expression of all helper T cellrelated genes in a dose-dependent manner, while $I L-4$ expression was significantly decreased at all doses. These findings are consistent with results of our previous study, which showed the suppression of Th-1, Th-2, and Th-17 cell-related splenic cytokine production and mRNA expression due to FA exposure in a dose dependent manner ${ }^{[46]}$. Furthermore, Wei et al. also reported that helper $T$ cell-related cytokines were suppressed in FA-exposed C57BL/6 mice ${ }^{[29]}$. Recent studies have revealed that FA exposure suppressed the Th1- and Th2-related cytokines in rodent models with ovalbumin sensitization, thereby resulting in a decrease in airway inflammation and bronchial hyper-responsiveness ${ }^{[27,30]}$. These results indicate that FA exposure suppressed effector T cell activity, inducing decreased T cell-related mRNA expression and cytokine production.

Treg cells actively suppress pathological and physiological immune responses, which contribute to the maintenance of immunological self-tolerance and immune homeostasis ${ }^{[55]}$. The suppressive functions of Treg cells can be grouped into four modes of action: 1) suppression mediated by the cytokines IL-10, IL-35, and TGF- $\beta$; 2) suppression by cytolysis mediated by Granzyme A or B; 3) suppression by metabolic disruption mediated by high-affinity CD25 and cyclic AMP; and 4) suppression by targeting dendritic cells through LAG3 and CTLA4 ${ }^{[56]}$. Hence, to determine whether FA exposure suppresses immune responses via Treg cells, we evaluated the population of helper T cells and Treg cells and evaluated the changes in their signalling pathways. Our results show that exposure to FA precipitated no difference in the population percentage of $\mathrm{CD} 4^{+}$helper T cells in minipigs. However, exposure to FA significantly increased the population size of splenic $\mathrm{CD} 4^{+} \mathrm{Foxp}^{+}$Treg cells. Thus, our findings were consistent with those of prior studies on FA-exposed human and rodent models.

Recent studies have reported that the NFAT-mediated signalling pathway contributes to the induction of Foxp3 expression, which controls the differentiation and function of Treg cells ${ }^{[42]}$. NFAT proteins are activated by cell surface receptors that are coupled to $\mathrm{Ca}^{2+}$ mobilization ${ }^{[57]}$. The increased levels of cytosolic calcium are bound by calmodulin, which in turn activates calcineurin - a calcium and calmodulin dependent serine/threonine protein phosphatase ${ }^{[58]}$. NFAT proteins are dephosphorylated by activated calcineurin, resulting in nuclear translocation of these proteins and the induction of NFATmediated gene transcription ${ }^{[59]}$. Recent in vivo and in vitro studies have revealed that NFAT1 plays a 
crucial role in the suppressive function of Treg cells ${ }^{[60-63]}$, along with enhancing and maintaining stable Foxp3 expression ${ }^{[58,64-67]}$. On the other hand, NFAT2 induces the activation of effector T cells and the production of effector cytokines in the immune system ${ }^{[68-70]}$. Our results show that exposure to FA resulted in an increase in NFAT1 expression and increase in Treg cell population size in minipigs, coupled with a decline in IL-4 production. In contrast, FA exposure precipitated no notable difference in NFAT2 expression. These results indicate that FA exposure activated the NFAT-mediated TCR signalling pathway with divergent expression of NFAT1 and NFAT2, leading to an increase in the population of Treg cells. These events may have subsequently induced an immunosuppressive microenvironment along with inhibition of effector T cell activity.

Owing to their close sequence homology with humans, minipigs are considered a useful non-rodent animal model platform for conducting toxicology research. In this study, we evaluated the effects of FA exposure on splenic immune responses in Yucatan minipigs. Our results revealed that exposure to FA increased the differentiation of Treg cells via the NFAT-mediated TCR signalling pathway with divergent expression of NFAT1 and NFAT2, resulting in the suppression of effector T cell activity with decreased production of T cell-related cytokines. Although some studies have reported that FA exposure may provoke or exacerbate the Th2-type responses in murine and human models, other studies have found that FA exposure dose not aggravate allergic responsiveness, nor does FA exposure reduce the development of allergic lung inflammation. The differences in species and strains of animal, concentrations and durations of FA exposure, and experimental protocols result in disparate immune responses being observed in response to FA exposure. Therefore, further studies under various conditions (28 days, or 90 days for long-term study, with administration of low and high doses of FA) are necessary to determine the impact of FA exposure on the immune systems. In conclusion, our findings provide insight into the molecular mechanisms underlying the FA-exposure-induced development of an immunosuppressive microenvironment, characterized by increased Foxp3 ${ }^{+}$Treg cell population. Development of such an immunosuppressive microenvironment may potentially result in detrimental health effects, such as increasing host susceptibility to opportunistic infections and the progression of cancer.

\section{Declarations}

\section{Data availability}

All data generated or analysed during the current study are accessible from the corresponding author upon reasonable request.

\section{Acknowledgments}

This research was supported by Grant number KK-1911 and KK-2109-01 from the Korea Institute of Toxicology. 


\section{Author contributions}

J. P. contributed to the experimental design, performed all the experiments and statistical analysis, and wrote the manuscript. G. K. contributed to the experimental design of FA exposure of minipig mask type inhalation system and animal management. Y. K. and J. L aided in the FA exposure of minipig mask type inhalation system and the western blot-related experiments. J. S. aided in the FA exposure of minipig mask type inhalation system and primary cell culture-related experiments. J. H. conceived the study, designed the experiments, and supervised J. P. All authors read and approved the final version of the manuscript.

\section{Competing interests}

The authors declare that they have no competing interests.

\section{References}

1. Bode, G. et al. The utility of the minipig as an animal model in regulatory toxicology. J. Pharmacol. Toxicol. Methods, 62.3, 196-220 (2002).

2. Svendsen, O. The minipig in toxicology. Exp. Toxicol. Pathol, 57 (5-6), 335-339 (2006).

3. Lacoste, L. et al. Intranasal midazolam in piglets: pharmacodynamics $(0.2 \mathrm{vs} 0.4 \mathrm{mg} / \mathrm{kg})$ and pharmacokinetics (0.4 mg/kg) with bioavailability determination. Lab Anim, 34 (1), 29-35 (2000).

4. Thing Mortensen, J., Brinck, P. \& Lichtenberg, J. The minipig in dermal toxicology. A literature review. Scand. J. Lab. Anim. Sci, 25, 77-84 (1998).

5. Forster, R. et al. The minipig as a platform for new technologies in toxicology. J. Pharmacol. Toxicol. Methods, 62 (3), 227-235 (2010).

6. Jørgensen, F. G. et al. Comparative analysis of protein coding sequences from human, mouse and the domesticated pig. BMC Biol, 3 (1), 1-15 (2005).

7. Wernersson, R. et al. Pigs in sequence space: a $0.66 \mathrm{X}$ coverage pig genome survey based on shotgun sequencing. BMC Genomics, 6, 70 (2005).

8. Rozkot, M., Václavková, E. \& Bělková, J. Minipigs as laboratory animals-review. Res. Pig Breed, 9 (2), 10-14 (2015).

9. Whyte, J. J. \& Prather, R. S. Genetic modifications of pigs for medicine and agriculture. Mol. Reprod. Dev, 78 (10-11), 879-891 (2011).

10. Fornhem, C. et al. Effects of local and systemic budesonide on allergen-induced airway reactions in the pig. Br. J. Pharmacol, 118 (4), 989-997 (1996).

11. Strüber, M. et al. Inhaled nitric oxide as a prophylactic treatment against reperfusion injury of the lung. Thorac. Cardiovasc. Surg, 47 (03), 179-182 (1999). 
12. Maggiorini, M. et al. Pulmonary vascular resistance in dogs and minipigs-effects of hypoxia and inhaled nitric oxide. Respir. Physiol, 111 (2), 213-222 (1998).

13. Hensel, A. et al. A porcine aerosol infection model for studying dose dependent effects caused by Actinobacillus pleuropneumoniae bacteria. J. Aerosol. Med, 6 (2), 73-88 (1993).

14. Kilburn, K. H. Indoor air effects after building renovation and in manufactured homes. Am. J. Med. Sci, 320 (4), 249-254 (2000).

15. Ezratty, V. et al. Effect of formaldehyde on asthmatic response to inhaled allergen challenge. Environ. Health Perspect, 115 (2), 210-214 (2007).

16. Carlson, R. M., Smith, M. C. \& Nedorost, S. T. Diagnosis and treatment of dermatitis due to formaldehyde resins in clothing. Dermatitis, 15 (4), 169-175 (2004).

17. Lino-dos-Santos-Franco, A. et al. Reduced allergic lung inflammation in rats following formaldehyde exposure: long-term effects on multiple effector systems. Toxicology, 256 (3), 157-163 (2009).

18. Songur, A. et al. The effects of the inhaled formaldehyde during the early postnatal period in the hippocampus of rats: a morphological and immunohistochemical study. Neurosci. Res. Commun, 33 (3), 168-178 (2003).

19. Baan, R. et al. A review of human carcinogens - part F: chemical agents and related occupations. Lancet Oncol, 10 (12), 1143-1144 (2009).

20. Kleinnijenhuis, A. J. et al. The determination of exogenous formaldehyde in blood of rats during and after inhalation exposure. Food Chem. Toxicol, 52, 105-112 (2013).

21. US EPA (United States Environmental Protection Agency). "Toxicological review of FormaldehydeInhalation assessment." (2010).

22. Salonen, H. et al. Volatile organic compounds and formaldehyde as explaining factors for sensory irritation in office environments. J. Occup. Environ. Hyg, 6 (4), 239-247 (2009).

23. Kim, K-H., Jahan, S. A. \& Lee, J. T. Exposure to formaldehyde and its potential human health hazards. J. Environ. Sci. Health C Environ. Carcinog. Ecotoxicol. Rev, 29 (4), 277-299 (2011).

24. McGregor, D. et al. Formaldehyde and glutaraldehyde and nasal cytotoxicity: case study within the context of the 2006 IPCS Human Framework for the Analysis of a cancer mode of action for humans. Crit. Rev. Toxicol, 36 (10), 821-835 (2006).

25. Sul, D. et al. Gene expression profiling in lung tissues from rats exposed to formaldehyde. Arch. Toxicol, 81 (8), 589-597 (2007).

26. Thompson, C. M. \& Grafström, R. C. Considerations for the implausibility of leukemia induction by formaldehyde. Toxicol. Sci, 120 (1), 230-232 (2011).

27. Li, L. et al. Differential effects of formaldehyde exposure on airway inflammation and bronchial hyperresponsiveness in BALB/c and C57BL/6 mice. PLoS One, 12 (6), e0179231 (2017).

28. Liu, D. et al. Adjuvant effects of gaseous formaldehyde on the hyper-responsiveness and inflammation in a mouse asthma model immunized by ovalbumin. J. Immunotoxicol, 8 (4), 305-314 (2011). 
29. Wei, H. et al. Aberrant production of Th1/Th2/Th17-related cytokines in serum of C57BL/6 mice after short-term formaldehyde exposure. Int. J. Environ. Res. Public Health, 11 (10), 10036-10050 (2014).

30. Fujimaki, H. et al. Differential immunogenic and neurogenic inflammatory responses in an allergic mouse model exposed to low levels of formaldehyde. Toxicology, 197 (1), 1-13 (2004).

31. Lino-dos-Santos-Franco, A. et al. Formaldehyde inhalation reduces respiratory mechanics in a rat model with allergic lung inflammation by altering the nitric oxide/cyclooxygenase-derived products relationship. Food Chem. Toxicol, 59, 731-738 (2013).

32. Jung, W-W. et al. Formaldehyde exposure induces airway inflammation by increasing eosinophil infiltrations through the regulation of reactive oxygen species production. Environ. Toxicol. Pharmacol, 24 (2), 174-182 (2007).

33. Hosgood, H. D. III et al. Occupational exposure to formaldehyde and alterations in lymphocyte subsets. A. J. Ind. Med, 56 (2), 252-257 (2013).

34. Sandikci, M., Eren, U. \& Kum, S. Effects of formaldehyde and xylene on CD4-and CD8-positive T cells in bronchus-associated lymphoid tissue in rats. Toxicol. Ind. Health, 23 (8), 471-477 (2007).

35. Aydin, S. et al. Assessment of immunotoxicity and genotoxicity in workers exposed to low concentrations of formaldehyde. Arch. Toxicol, 87 (1), 145-153 (2013).

36. Nakayamada, S. et al. Helper T cell diversity and plasticity. Curr. Opin. Immunol, 24 (3), $297-302$ (2012).

37. Gagliani, N. \& Huber, S. Basic aspects of T helper cell differentiation. Methods Mol. Biol, 1514, 19-30 (2017).

38. Hirahara, K. et al. Mechanisms underlying helper T-cell plasticity: implications for immune-mediated disease. J. Allergy Clin. Immunol, 131 (5), 1276-1287 (2013).

39. Saravia, J., Chapman, N. M. \& Chi, H. Helper T Cell Differentiation. Cell. Mol. Immunol, 16 (7), $634-$ 643 (2019).

40. Cosmi, L. et al. T helper cells plasticity in inflammation. Cytometry A, 85 (1), 36-42 (2014).

41. Zhou, L., Chong, M. M. W. \& Littman, D. R. Plasticity of CD4 + T cell lineage differentiation., 30 (5), 646-655 (2009).

42. Huehn, J., Polansky, J. K. \& Hamann, A. Epigenetic control of FOXP3 expression: the key to a stable regulatory T-cell lineage? Nat. Rev. Immunol, 9 (2), 83-89 (2009).

43. Cavassani, K. A. et al. The post sepsis-induced expansion and enhanced function of regulatory $T$ cells create an environment to potentiate tumor growth., 115 (22), 4403-4411 (2010).

44. Gera, R. et al. Arsenic exposure impels CD4 commitment in thymus and suppress T cell cytokine secretion by increasing regulatory T cells. Sci. Rep, 7 (1), 1-13 (2017).

45. Smith, A. H. et al. Evidence from Chile that arsenic in drinking water may increase mortality from pulmonary tuberculosis. Am. J. Epidemiol, 173 (4), 414-420 (2011).

46. Park, J. et al. Formaldehyde exposure induces regulatory $T$ cell-mediated immunosuppression via calcineurin-NFAT signalling pathway. Sci. Rep, 10 (1), 1-10 (2020). 
47. Lino-dos-Santos-Franco, A. et al. Differential effects of formaldehyde exposure on the cell influx and vascular permeability in a rat model of allergic lung inflammation. Toxicol. Lett, 197 (3), 211-218 (2010).

48. Organisation for Economic Co-operation and Development (OECD). "SIDS initial assessment report for SIAM 14: Formaldehyde." (2002).

49. Regulations, O. S. H. A. "formaldehyde - 1910.1048 Occupational Safety (\& Health Administration." US Department of Labor, 1992).

50. Kim, E-M. Formaldehyde exposure impairs the function and differentiation of NK cells. Toxicol. Lett, 223 (2), 154-161 (2013).

51. Liu, Q. et al. Formaldehyde inhalation triggers autophagy in rat lung tissues. Toxicol Ind Health, 34 (12), 834-841 (2018).

52. Ohtsuka, R. et al. Rat strain difference in histology and expression of Th1-and Th2-related cytokines in nasal mucosa after short-term formaldehyde inhalation. Exp. Toxicol. Pathol, 54 (4), 287-291 (2003).

53. Mebius, R. E. \& Kraal, G. Structure and function of the spleen. Nat. Rev. Immunol, 5 (8), 606-616 (2005).

54. Lee, C-G., Sahoo, A. \& Im, S-H. Epigenetic regulation of cytokine gene expression in T lymphocytes. Yonsei Med. J, 50 (3), 322 (2009).

55. Miyara, M. \& Sakaguchi, S. Natural regulatory T cells: mechanisms of suppression. Trends Mol. Med, 13 (3), 108-116 (2007).

56. Vignali, D. A. A., Collison, L. W. \& Workman, C. J. How regulatory T cells work. Nat. Rev. Immunol, 8 (7), 523-532 (2008).

57. Müller, M. R. \& Rao, A. NFAT, immunity and cancer: a transcription factor comes of age. Nat. Rev. Immunol, 10 (9), 645-656 (2010).

58. Vaeth, M. \& Feske, S. NFAT control of immune function: New Frontiers for an Abiding Trooper. F1000Res, 2 (7), 260 (2018).

59. Macian, F. NFAT proteins: key regulators of T-cell development and function. Nat. Rev. Immunol, 5 (6), 472-484 (2005).

60. Erb, K. J. et al. Mice deficient in nuclear factor of activated T-cell transcription factor c2 mount increased Th2 responses after infection with Nippostrongylus brasiliensis and decreased Th1 responses after mycobacterial infection. Infect. Immun, 71 (11), 6641-6647 (2003).

61. Hodge, M. R. et al. Hyperproliferation and dysregulation of IL-4 expression in NF-ATp-deficient mice. Immunity, 4 (4), 397-405 (1996).

62. Viola, J. P. et al. Regulation of allergic inflammation and eosinophil recruitment in mice lacking the transcription factor NFAT1: role of interleukin-4 (IL-4) and IL-5. Blood, 91 (7), 2223-2230 (1998).

63. Xanthoudakis, S. et al. An enhanced immune response in mice lacking the transcription factor NFAT1. Science, 272 (5263), 892-895 (1996). 
64. Wu, Y. et al. FOXP3 controls regulatory T cell function through cooperation with NFAT. Cell, 126 (2), 375-387 (2006).

65. Gupta, S. et al. Differential requirement of PKC- $\theta$ in the development and function of natural regulatory T cells. Mol. Immunol, 46 (2), 213-224 (2008).

66. Mantel, P-Y. et al. Molecular mechanisms underlying FOXP3 induction in human T cells. J. Immunol, 176 (6), 3593-3602 (2006).

67. Bopp, T. et al. NFATc2 and NFATc3 transcription factors play a crucial role in suppression of CD4 + T lymphocytes by CD4 + CD25 + regulatory T cells. J. Exp. Med, 201 (2), 181-187 (2005).

68. Gomez-Rodriguez, J. et al. Differential expression of interleukin-17A and-17F is coupled to T cell receptor signaling via inducible T cell kinase. Immunity, 31 (4), 587-597 (2009).

69. Ranger, A. M. et al. Delayed lymphoid repopulation with defects in IL-4-driven responses produced by inactivation of NF-ATC., 8 (1), 125-134 (1998).

70. Yoshida, H. et al. The transcription factor NF-ATc1 regulates lymphocyte proliferation and Th2 cytokine production. Immunity, 8 (1), 115-124 (1998).

\section{Figures}

Figure 1.

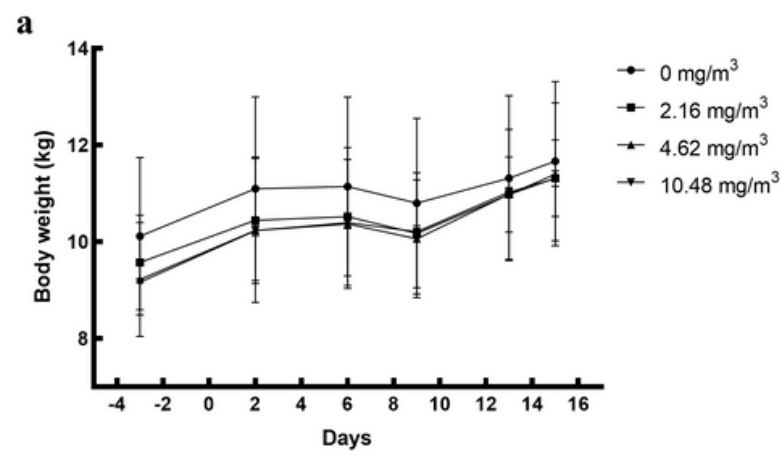

b

e

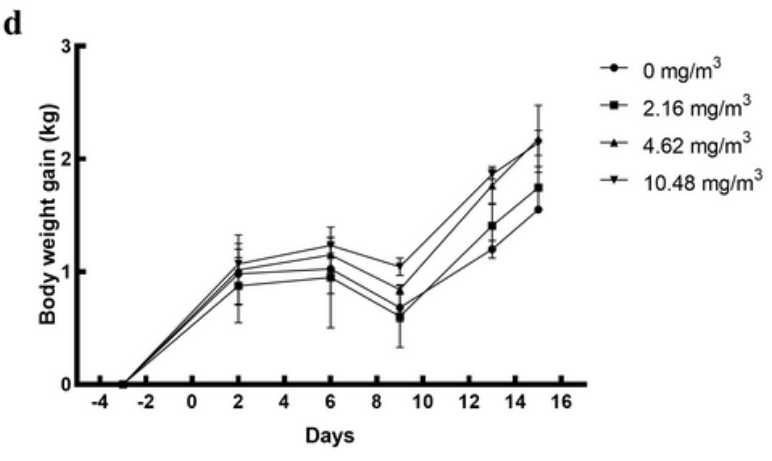

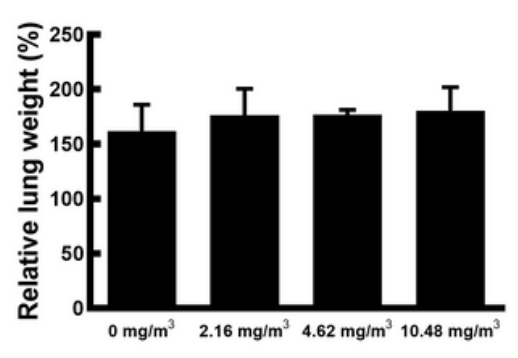

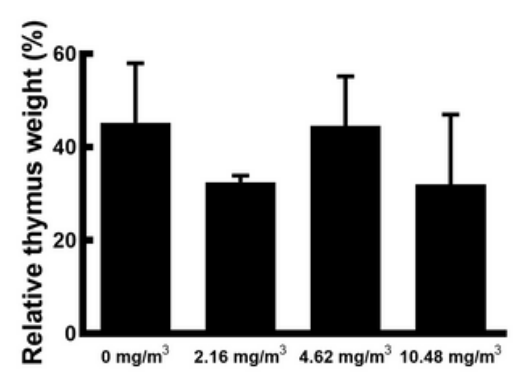

c

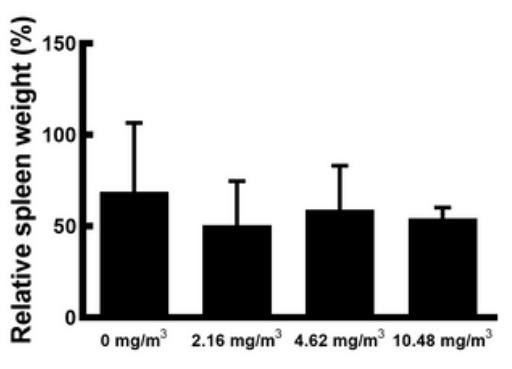

\section{Figure 1}

Body weight and weight of organs in Yucatan minipigs. Changes in body weight (a) and weight gain (d) of minipigs exposed to FA. Relative weights of organs, including the lung (b), spleen (c), and thymus (e) 
were calculated. Data are presented as the mean $\pm S D$ ( $n=2$ minipigs/group).

Figure 2

a

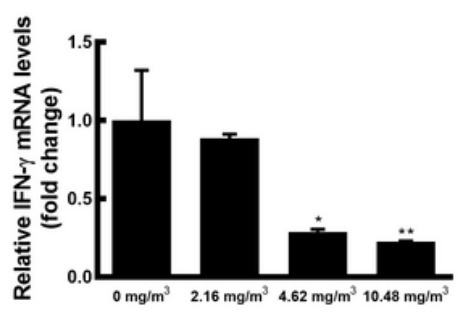

d

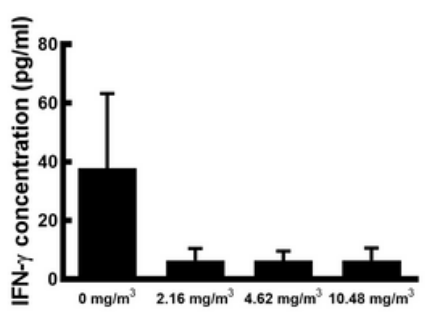

b

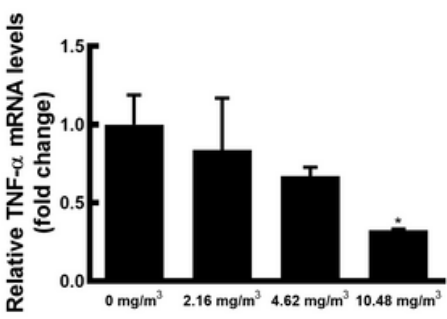

e

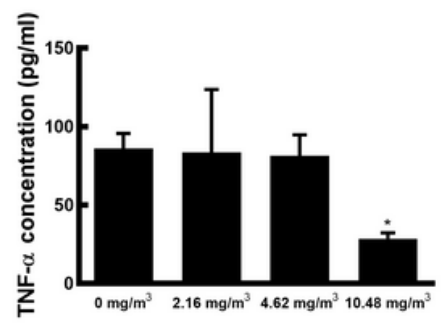

c

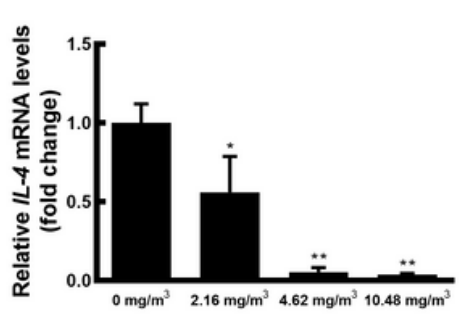

f

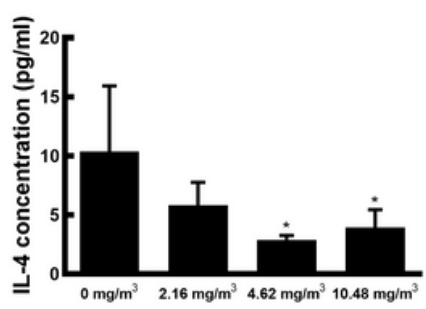

Figure 2

Helper T cell-related mRNA expression and cytokine production by FA exposure. Splenocytes isolated from FA exposed minipigs were cultured with $2.5 \mu \mathrm{g} / \mathrm{mL}$ Con A for $72 \mathrm{~h}$. The mRNA expression of genes associated with Th1 (IFN- $y$, TNF-a) and Th2 (IL-4) were evaluated by qRT-PCR (a-c). Target gene expression was normalized using GAPDH expression and the expression was presented as fold change relative to the control group. Production of cytokines associated with Th1 (IFN- $y$, TNF-a) and Th2 (IL-4) were evaluated (d-f). Data are presented as the mean $\pm S D\left(n=2\right.$ minipigs/group). ${ }^{\star} p<0.05,{ }^{\star \star} p<0.01$. 
Figure 3

a

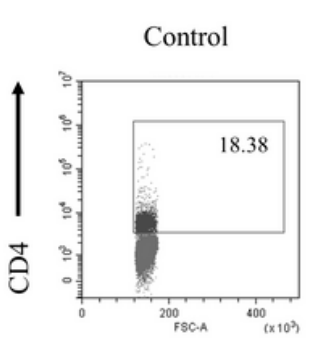

FSC

b
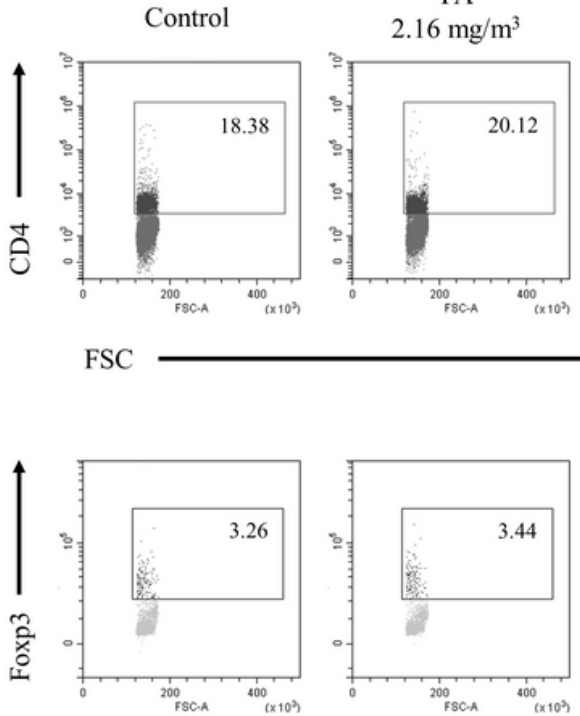

FA
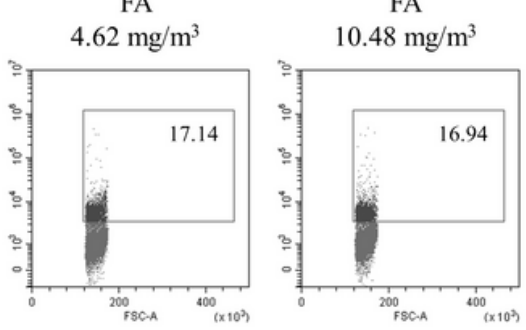

FSC
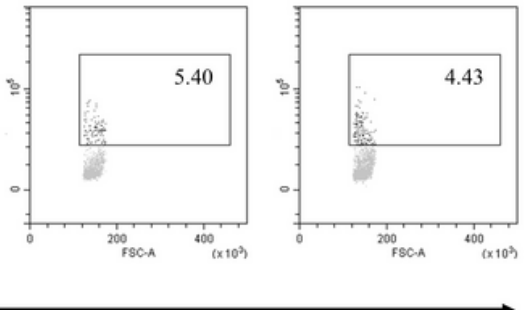
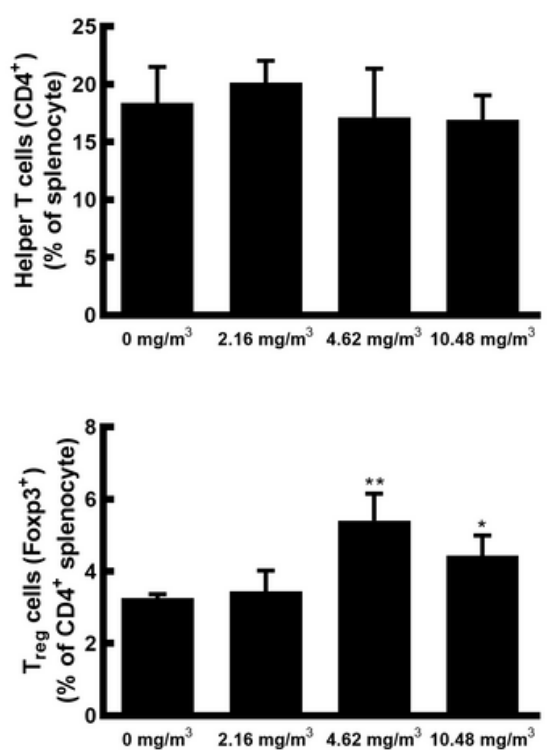

\section{Figure 3}

Regulation of the helper T cell and Treg cell populations by FA exposure. Single spleen cells isolated from minipigs exposed to FA were stained with PC 5.5-conjugated anti-CD4 and FITC-conjugated anti-Foxp3 monoclonal antibodies. The fluorescence levels of antibody were measured using a CytoFlex flow cytometer. (a) CD4+ helper T cell population in single spleen cells. (b) CD4+ Foxp3+ Treg cell population in CD4+ helper T cells. Data are expressed as representative dot plots and presented as the mean \pm SD ( $\mathrm{n}$ $=2$ minipig/group). ${ }^{\star} p<0.05,{ }^{\star *} p<0.01$. 
Figure 4

a
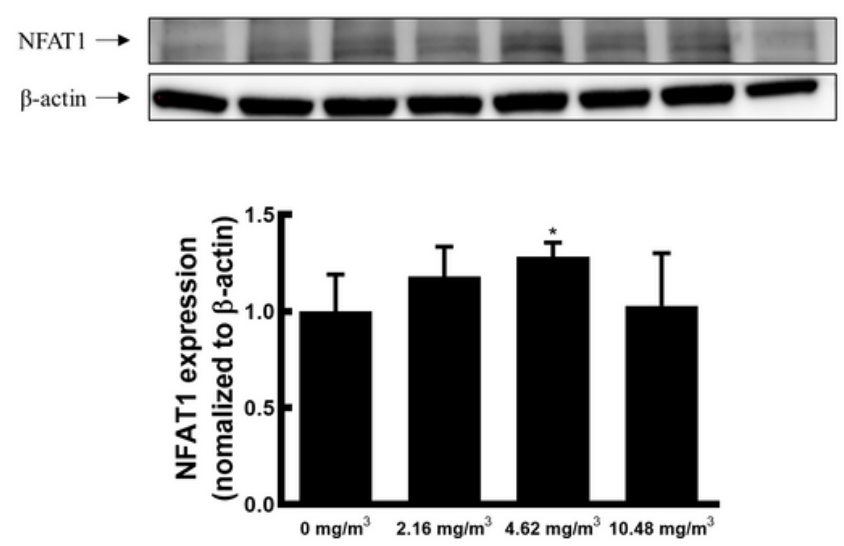

b
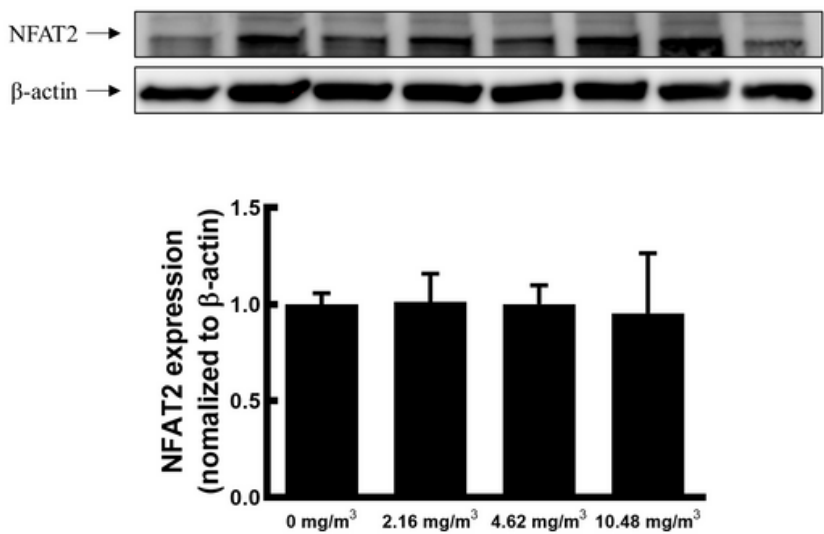

\section{Figure 4}

Expression of NFAT1 and NFAT2 by FA exposure in the spleen. (a) NFAT1 and NFAT2 expression assessed by western blotting. (b) Densitometrical analysis of the blots. The target protein expression levels were normalized to $\beta$-actin expression. Data are presented as the mean \pm SD $(n=2$ minipigs/group). ${ }^{\star} \mathrm{p}<0.05$. 
Figure 5

a

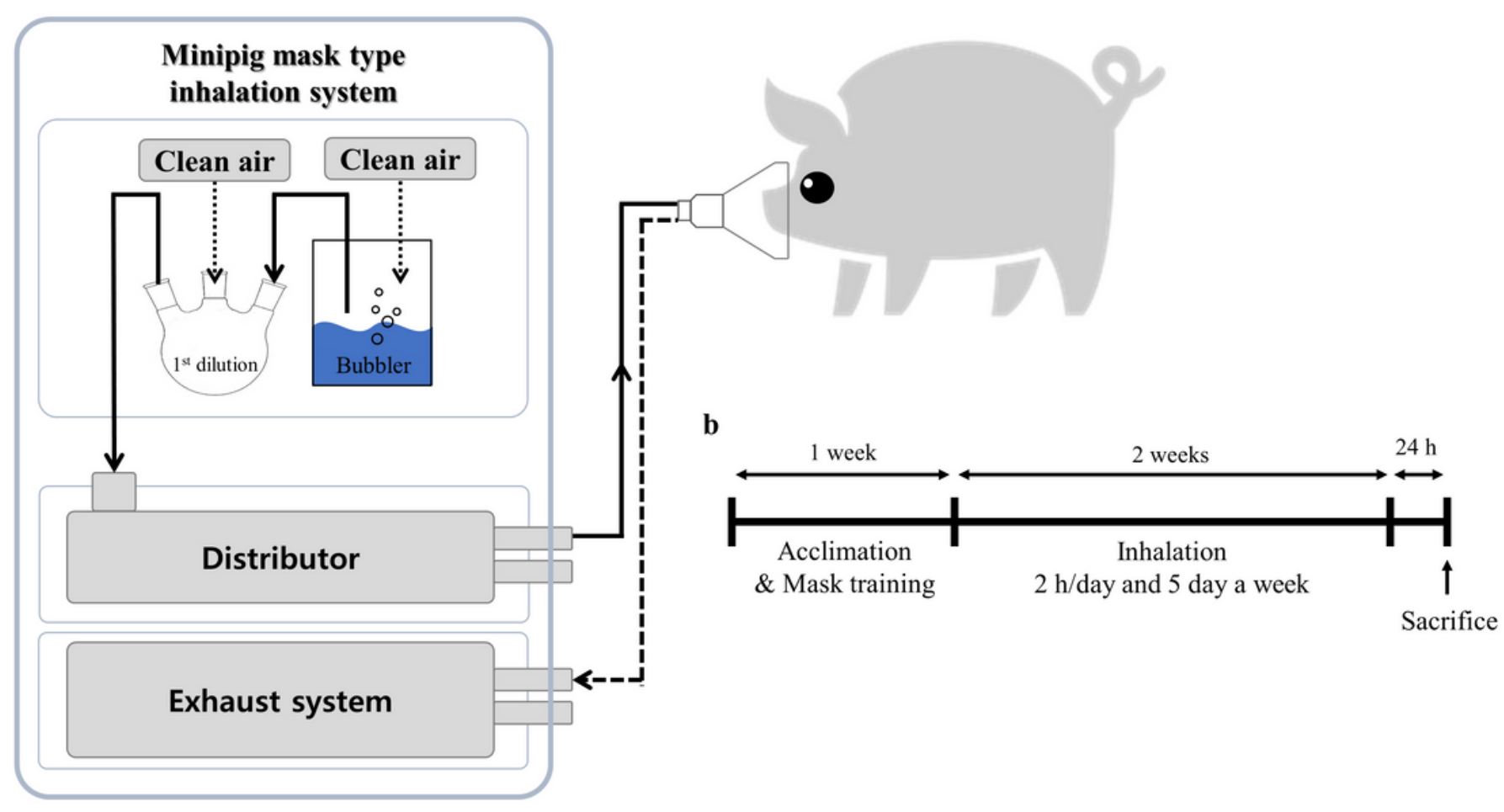

Figure 5

Schematic of the minipig mask type inhalation system for FA exposure and experimental design for assessing the effect of FA exposure on minipigs. (a) FA was generated using a gas bubbler and diluted with clean air using a multi-neck flask. (b) All minipigs received mask training for $2 \mathrm{~h}$ a day before FA exposure. Minipigs in the treatment groups were exposed to FA for a period of 2 weeks at $2 \mathrm{~h} /$ day, 5 days a week using a minipig mask type inhalation system. 
Figure 6

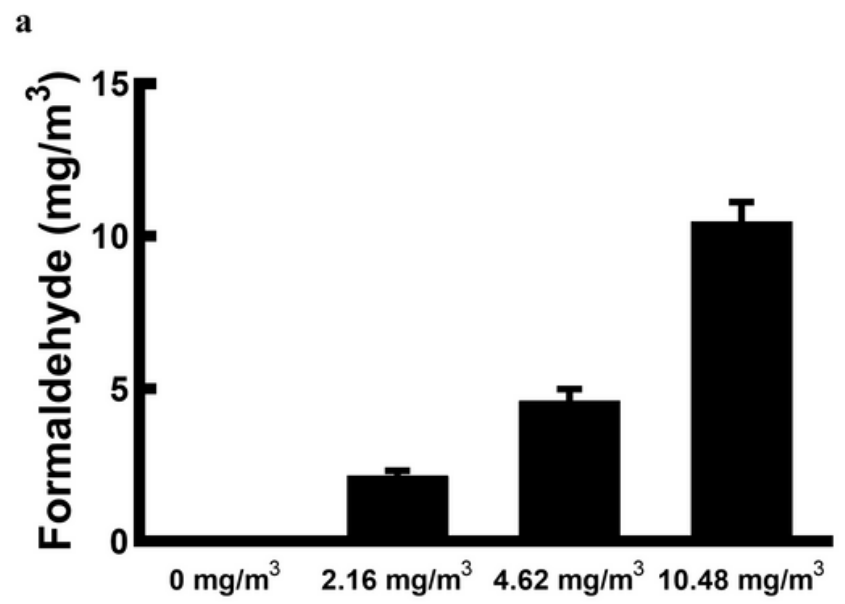

b

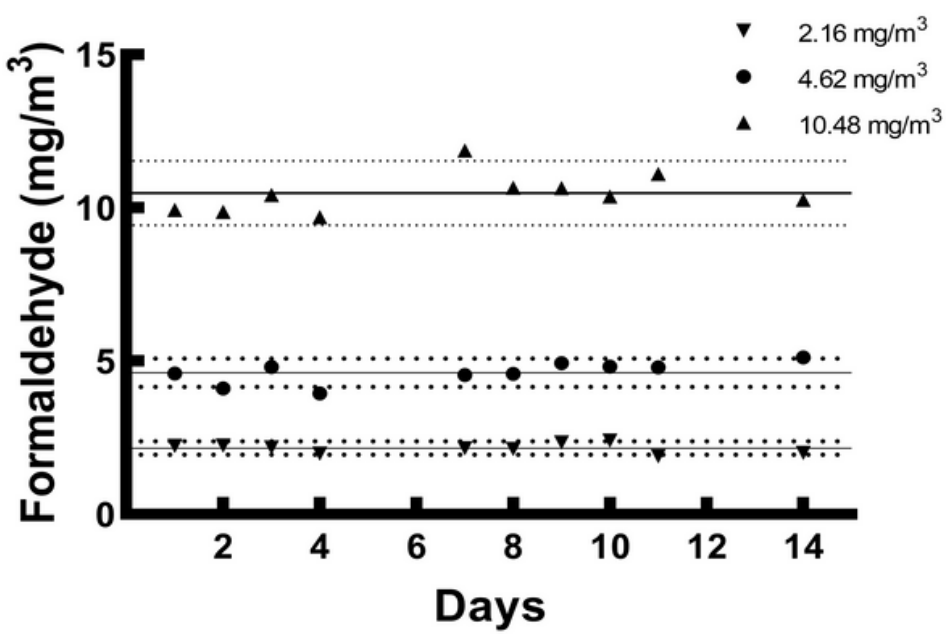

Figure 6

FA doses assessed in the study. The (a) mean and (b) daily concentrations of FA exposure were monitored using a Top Solid DNPH cartridge and HPLC system. The groups of Yucatan minipigs were exposed to $0 \mathrm{mg} / \mathrm{m} 3$ (control), $2.16 \pm 0.16 \mathrm{mg} / \mathrm{m} 3,4.62 \pm 0.36 \mathrm{mg} / \mathrm{m} 3$, or $10.48 \pm 0.64 \mathrm{mg} / \mathrm{m} 3 \mathrm{FA}$ for 2 weeks at $2 \mathrm{~h} /$ day and 5 days/week. Data are presented as the mean \pm SD. 